PROCEEDINGS OF THE AMERICAN MATHEMATICAL SOCIETY

Volume 135, Number 5, May 2007, Pages 1389-1397

S 0002-9939(06)08590-X

Article electronically published on October 27, 2006

\title{
CHARACTERIZATIONS OF POSITIVE SELFADJOINT EXTENSIONS
}

\author{
ZOLTÁN SEBESTYÉN AND JAN STOCHEL
}

(Communicated by Joseph A. Ball)

Dedicated to Henk de Snoo on the occasion of his sixtieth birthday.

\begin{abstract}
The set of all positive selfadjoint extensions of a positive operator $T$ (which is not assumed to be densely defined) is described with the help of the partial order which is relevant to the theory of quadratic forms. This enables us to improve and extend a result of M. G. Krein to the case of not necessarily densely defined operators $T$.
\end{abstract}

1.

In the present paper we continue our investigations of the extension problem we started in [19] (see [1] for the case of closed positive operators and [16, 17, 21] for the case of bounded positive operators; see also [12, 6, 5, 13, 14, 2, 20, 18, 3, 4, for related papers). Our aim is to describe the set of all positive selfadjoint extensions of a given positive operator (not necessarily densely defined) using the language of quadratic forms.

Throughout what follows, $\mathcal{H}$ stands for a complex Hilbert space. By an operator in $\mathcal{H}$ we understand a linear mapping $A: \mathcal{H} \supseteq \mathcal{D}(A) \rightarrow \mathcal{H}$ defined on a linear subspace $\mathcal{D}(A)$ of $\mathcal{H}$, called the domain of $A ; \mathcal{N}(A)$ and $\mathcal{R}(A)$ stand for the kernel and the range of $A$, respectively. If $A$ and $B$ are operators in $\mathcal{H}$ such that $\mathcal{D}(A) \subseteq$ $\mathcal{D}(B)$ and $A h=B h$ for all $h \in \mathcal{D}(A)$, then we say that $B$ extends $A$ and write $A \subseteq B$. If $A$ is a densely defined operator in $\mathcal{H}$, then its adjoint is denoted by $A^{*}$. A densely defined operator $A$ in $\mathcal{H}$ is called selfadjoint if $A=A^{*}$. We write $\boldsymbol{B}(\mathcal{H})$ for the $C^{*}$-algebra of all bounded operators in $\mathcal{H}$ whose domains are equal to $\mathcal{H}$.

An operator $A$ in $\mathcal{H}$ is said to be positive if $\langle A h, h\rangle \geqslant 0$ for all $h \in \mathcal{D}(A)$ (we do not assume $A$ to be densely defined). Positive operators may not be closable. If $A$ is a positive selfadjoint operator in $\mathcal{H}$, then there exists a unique positive selfadjoint operator $X$ in $\mathcal{H}$ solving the equation $A=X^{2}$ (cf. [11, 17); such $X$ is usually denoted by $A^{1 / 2}$. If $A$ and $B$ are positive selfadjoint operators in $\mathcal{H}$ which satisfy the condition

$$
\mathcal{D}\left(B^{1 / 2}\right) \subseteq \mathcal{D}\left(A^{1 / 2}\right) \text { and }\left\|A^{1 / 2} h\right\|^{2} \leqslant\left\|B^{1 / 2} h\right\|^{2} \text { for all } h \in \mathcal{D}\left(B^{1 / 2}\right),
$$

Received by the editors June 30, 2005 and, in revised form, November 29, 2005.

2000 Mathematics Subject Classification. Primary 47A20, 47B25; Secondary 47A63.

Key words and phrases. Positive operator, selfadjoint operator, positive selfadjoint extension, Krein-von Neumann extension, Friedrichs extension.

The research of the second author was supported by KBN grant 2 P03A 037024.

(C)2006 American Mathematical Society Reverts to public domain 28 years from publication 
then we write $A \prec B$. The relation $\prec$ is a partial order on the set of all positive selfadjoint operators in $\mathcal{H}$. In particular, the following property of $\prec$ turns out to be useful:

$$
\text { if } A \prec B \text { and } B \prec A \text {, then } A=B \text {. }
$$

The reader should be aware of the fact that, in general, $A \prec B$ does not coincide with $A \leqslant B$, where $A \leqslant B$ means that $\mathcal{D}(A) \subseteq \mathcal{D}(B)$ and $\langle A h, h\rangle \leqslant\langle B h, h\rangle$ for all $h \in \mathcal{D}(A)$. However, the relations $\prec$ and $\leqslant$ coincide on the set of all bounded positive selfadjoint operators on $\mathcal{H}$. Recall that according to [2, Lemma 2.1], $A \prec B$ if and only if

$$
\mathcal{D}(B) \subseteq \mathcal{D}\left(A^{1 / 2}\right) \text { and }\left\|A^{1 / 2} h\right\|^{2} \leqslant\langle B h, h\rangle \text { for all } h \in \mathcal{D}(B) .
$$

One can also show that $A \prec B$ if and only if there exists a contraction $C \in$ $\boldsymbol{B}(\mathcal{H})$ such that $C B^{1 / 2} \subseteq A^{1 / 2}$ (consequently $C B^{1 / 2} \subseteq A^{1 / 2} \subseteq B^{1 / 2} C^{*}$, which implies that $C B^{1 / 2}$ is a symmetric operator). The contraction $C$ can always be chosen so that $\mathcal{N}(B) \subseteq \mathcal{N}(C)$. This property determines $C$ uniquely. Another characterization of the relation $\prec$ can be written in terms of the resolvents of $A$ and $B$ as follows: $A \prec B$ if and only if $(B-x)^{-1} \leqslant(A-x)^{-1}$ for all real $x<0$ or equivalently for some real $x<0$ (cf. [11, page 330, Theorem 2.21]). For more details on this subject we refer the reader to [11] or [23].

2.

Given a positive operator $T$ in $\mathcal{H}$, we define

$$
\begin{aligned}
\operatorname{Ext}(T) & =\{S: S \text { is a positive selfadjoint operator in } \mathcal{H} \text { such that } T \subseteq S\} \\
\nu_{T}(f) & =\sup \left\{|\langle f, T g\rangle|^{2}: g \in \mathcal{D}(T),\langle T g, g\rangle \leqslant 1\right\}, \quad f \in \mathcal{H}, \\
\mathcal{D}_{*}[T] & =\left\{f \in \mathcal{H}: \nu_{T}(f)<\infty\right\}
\end{aligned}
$$

It may happen that $\operatorname{Ext}(T)$ is an empty set and $\mathcal{D}_{*}[T]$ is not dense in $\mathcal{H}$ (see [20] for explicit examples illustrating this phenomenon).

We now list some basic properties of the function $\nu_{T}: \mathcal{H} \rightarrow[0, \infty]$.

Proposition 1. Let $T$ be a positive operator in $\mathcal{H}$. Then

(1) $\mathcal{D}_{*}[T]$ is a linear subspace of $\mathcal{H}$,

(2) a vector $f \in \mathcal{H}$ is a member of $\mathcal{D}_{*}[T]$ if and only if there exists a constant $M \geqslant 0$ such that $|\langle f, T g\rangle|^{2} \leqslant M\langle T g, g\rangle$ for all $g \in \mathcal{D}(T)$; the least such constant $M$ is equal to $\nu_{T}(f)$,

(3) if $T$ is closable, then its closure $\bar{T}$ is positive, $\nu_{T}=\nu_{\bar{T}}$ and $\mathcal{D}_{*}[T]=\mathcal{D}_{*}[\bar{T}]$,

(4) if $\mathcal{D}_{*}[T]$ is dense in $\mathcal{H}$, then $T$ is closable 1

(5) $\mathcal{D}(T) \subseteq \mathcal{D}_{*}[T]$ and $\nu_{T}(g)=\langle T g, g\rangle$ for all $g \in \mathcal{D}(T)$,

(6) the function $\mathfrak{t}_{T}: \mathcal{H} \supseteq \mathcal{D}\left(\mathfrak{t}_{T}\right) \rightarrow \mathbb{C}$ defined by

\footnotetext{
${ }^{1}$ One can show more, namely that $T$ is positively closable; cf. [1, Corollary 1].
} 


$$
\mathcal{D}\left(\mathfrak{t}_{T}\right)=\mathcal{D}_{*}[T] \quad \text { and } \quad \mathfrak{t}_{T}[f]=\nu_{T}(f) \text { for } f \in \mathcal{D}\left(\mathfrak{t}_{T}\right)
$$

is a closed positive quadratic form $2^{2}$ in $\mathcal{H}$.

Proof. The proof of (1) and (2) is straightforward. It follows from (2) that

$$
|\langle f, T g\rangle|^{2} \leqslant \nu_{T}(f)\langle T g, g\rangle, \quad f \in \mathcal{D}_{*}[T], g \in \mathcal{D}(T),
$$

which implies that $|\langle f, \bar{T} g\rangle|^{2} \leqslant \nu_{T}(f)\langle\bar{T} g, g\rangle$ for all $f \in \mathcal{D}_{*}[T]$ and $g \in \mathcal{D}(\bar{T})$. This leads to $\nu_{\bar{T}} \leqslant \nu_{T}$. Since the reverse inequality is obvious, the proof of (3) is complete. For (4), take $\left\{g_{n}\right\}_{n=1}^{\infty} \subseteq \mathcal{D}(T)$ and $h \in \mathcal{H}$ such that $g_{n} \rightarrow 0$ and $T g_{n} \rightarrow h$ as $n \rightarrow \infty$. Then, by (3), $h \perp \mathcal{D}_{*}[T]$, which yields $h=0$. Hence $T$ is closable.

(5) Applying the Schwarz inequality to the positive definite sesquilinear form $\mathcal{D}(T) \times \mathcal{D}(T) \ni(f, g) \mapsto\langle T f, g\rangle \in \mathbb{C}$, we see that $g \in \mathcal{D}_{*}[T]$ and $\nu_{T}(g) \leqslant\langle T g, g\rangle$ for all $g \in \mathcal{D}(T)$. If $g \in \mathcal{D}(T)$ and $\langle T g, g\rangle>0$, then $\tilde{g} \stackrel{\text { def }}{=} \frac{1}{\sqrt{\langle T g, g\rangle}} g \in \mathcal{D}(T),\langle T \tilde{g}, \tilde{g}\rangle=1$ and $|\langle g, T \tilde{g}\rangle|^{2}=\langle T g, g\rangle$, which implies that $\nu_{T}(g)=\langle T g, g\rangle$.

(6) Since the operator $T$ is positive, the set $\mathfrak{N}_{T}=\{g \in \mathcal{D}(T):\langle T g, g\rangle=0\}$ is a linear subspace of $\mathcal{D}(T)$, and the formula

$$
\left\langle f+\mathfrak{N}_{T}, g+\mathfrak{N}_{T}\right\rangle:=\langle T f, g\rangle, \quad f, g \in \mathcal{D}(T),
$$

defines an inner product on the quotient linear space $\mathcal{D}(T) / \mathfrak{N}_{T}$. Denote by $\mathcal{H}_{T}$ the completion of $\mathcal{D}(T) / \mathfrak{N}_{T}$ with respect to the so-defined inner product (this construction appears in [19]). It is now a matter of routine to show that a vector $f \in \mathcal{H}$ belongs to $\mathcal{D}_{*}[T]$ if and only if there exists a continuous linear functional $\xi_{f}$ on $\mathcal{H}_{T}$ such that

$$
\xi_{f}\left(g+\mathfrak{N}_{T}\right)=\langle T g, f\rangle, \quad g \in \mathcal{D}(T) .
$$

This in turn, by the Riesz representation theorem applied to the functional $\xi_{f}$, is equivalent to the existence of a (unique) vector $\widetilde{T} f \in \mathcal{H}_{T}$ such that

$$
\langle T g, f\rangle=\left\langle g+\mathfrak{N}_{T}, \widetilde{T} f\right\rangle, \quad g \in \mathcal{D}(T) .
$$

It follows from (41) and (5) that

$$
\nu_{T}(f)=\left\|\xi_{f}\right\|^{2}=\|\widetilde{T} f\|^{2}, \quad f \in \mathcal{D}_{*}[T] .
$$

By (5), the mapping $\widetilde{T}: \mathcal{D}_{*}[T] \longrightarrow \mathcal{H}_{T}$ is linear. In turn, (66) leads to

$$
\mathfrak{t}_{T}[f]=\langle\widetilde{T} f, \widetilde{T} f\rangle, \quad f \in \mathcal{D}_{*}[T],
$$

which shows that $\mathfrak{t}_{T}$ is a positive quadratic form in $\mathcal{H}$. To prove its closedness, take a sequence $\left\{f_{n}\right\}_{n=1}^{\infty} \subseteq \mathcal{D}\left(\mathfrak{t}_{T}\right)$ and a vector $f \in \mathcal{H}$ such that $f_{n} \rightarrow f$ as $n \rightarrow \infty$ and $\mathfrak{t}_{T}\left[f_{m}-f_{n}\right] \rightarrow 0$ as $m, n \rightarrow \infty$. It follows from (7) that $\left\|\widetilde{T}\left(f_{m}-f_{n}\right)\right\| \rightarrow 0$ as $m, n \rightarrow \infty$. Hence, there exists $h \in \mathcal{H}_{T}$ such that $\widetilde{T} f_{n} \rightarrow h$ as $n \rightarrow \infty$. Employing (5), we get

$$
\langle T g, f\rangle=\lim _{n \rightarrow \infty}\left\langle T g, f_{n}\right\rangle=\lim _{n \rightarrow \infty}\left\langle g+\mathfrak{N}_{T}, \widetilde{T} f_{n}\right\rangle=\left\langle g+\mathfrak{N}_{T}, h\right\rangle, \quad g \in \mathcal{D}(T) .
$$

This implies that $f \in \mathcal{D}\left(\mathfrak{t}_{T}\right)$ and $\widetilde{T} f=h$. Thus, by (7), $\mathfrak{t}_{T}\left[f_{n}-f\right] \rightarrow 0$ as $n \rightarrow \infty$. This means that the form $\mathfrak{t}_{T}$ is closed.

${ }^{2}$ We refer the reader to 11 for the foundations of the theory of quadratic forms including necessary terminology. 
3.

The following theorem is a recapitulation of [19, Theorem 1 and Corollary 3]. In view of Proposition 1, Theorem 2 can also be deduced from 1, Theorem 1 and Corollary 1], where closed positive operators are considered. For the reader's convenience, we sketch the proof of Theorem 2 focusing our attention on the last part of the conclusion (see [13, 14] for other variants of the proof).

Theorem 2. Let $T$ be a positive operator in $\mathcal{H}$. Then the following conditions are equivalent:

(i) $\operatorname{Ext}(T) \neq \varnothing$,

(ii) the linear space $\mathcal{D}_{*}[T]$ is dense in $\mathcal{H}$.

Moreover, if (i) holds, then there exists the smallest member $T_{\mathrm{N}}$ of $\operatorname{Ext}(T)$ with respect to the partial order $\prec$. The operator $T_{\mathrm{N}}$ is a unique positive selfadjoint operator in $\mathcal{H}$ satisfying the following two conditions:

$$
\begin{aligned}
\mathcal{D}\left(T_{\mathrm{N}}^{1 / 2}\right) & =\mathcal{D}_{*}[T], \\
\left\|T_{\mathrm{N}}^{1 / 2} f\right\|^{2} & =\nu_{T}(f), \quad f \in \mathcal{D}_{*}[T] .
\end{aligned}
$$

A sketch of the proof (see [19] for more details). Assume that $\operatorname{Ext}(T)$ is nonempty. Then there exists a complex Hilbert space $\mathcal{K}$ and a linear mapping $Q: \mathcal{H} \supseteq \mathcal{D}(T) \rightarrow$ $\mathcal{K}$ such that

$$
\begin{aligned}
\langle T f, g\rangle & =\langle Q f, Q g\rangle, \quad f, g \in \mathcal{D}(T), \\
\mathcal{K} & =\overline{Q(\mathcal{D}(T))} .
\end{aligned}
$$

Define the operator $V: \mathcal{K} \supseteq Q(\mathcal{D}(T)) \rightarrow \mathcal{H}$ by

$$
V(Q g)=T g, \quad g \in \mathcal{D}(T) .
$$

Its adjoint $V^{*}: \mathcal{H} \supseteq \mathcal{D}\left(V^{*}\right) \rightarrow \mathcal{K}$ is densely defined, and $T_{\mathrm{N}} \stackrel{\text { def }}{=} V^{* *} V^{*}$ is the smallest member of $\operatorname{Ext}(T)$. It follows from (10) and (12) that

$$
\nu_{T}(f)=\sup \left\{|\langle f, V(Q g)\rangle|^{2}: g \in \mathcal{D}(T),\|Q g\|^{2} \leqslant 1\right\}, \quad f \in \mathcal{H} .
$$

This, the definition of $V^{*}$ and (11) yield

$$
\mathcal{D}\left(V^{*}\right)=\left\{f \in \mathcal{H}: \nu_{T}(f)<\infty\right\} \text { and }\left\|V^{*} f\right\|^{2}=\nu_{T}(f) \text { for all } f \in \mathcal{D}\left(V^{*}\right) .
$$

Since $3 \mathcal{D}\left(V^{*}\right)=\mathcal{D}\left(T_{\mathrm{N}}^{1 / 2}\right)$ and $\left\|V^{*} f\right\|^{2}=\left\|T_{\mathrm{N}}^{1 / 2} f\right\|^{2}$ for all $f \in \mathcal{D}_{*}[T]$, we conclude that $T_{\mathrm{N}}$ satisfies the conditions (8) and (9).

The uniqueness of $T_{\mathrm{N}}$ satisfying (8) and (9) is a direct consequence of (1).

If the operator $T$ is densely defined, then the operator $T_{\mathrm{N}}$ defined in Theorem 2 is called the Krein-von Neumann extension of $T$ (cf. 24, 12]). Note that if $S$ is a positive selfadjoint operator in $\mathcal{H}$, then $\operatorname{Ext}(S)=\{S\}$. Thus Theorem 2 yields

$$
\mathcal{D}_{*}[S]=\mathcal{D}\left(S^{1 / 2}\right) \text { and }\left\|S^{1 / 2} f\right\|^{2}=\nu_{S}(f) \text { for all } f \in \mathcal{D}_{*}[S] \quad(S \text { selfadjoint }) .
$$

\section{4.}

In this section we give an example of the application of Theorem 2

\footnotetext{
${ }^{3}$ It is well known 7 that if $A: \mathcal{H} \supseteq \mathcal{D}(A) \longrightarrow \mathcal{K}$ is a closed densely defined operator (in our case $\left.A=V^{*}\right)$, then $\mathcal{D}\left(\left(A^{*} A\right)^{1 / 2}\right)=\mathcal{D}(A)$ and $\left\|\left(A^{*} A\right)^{1 / 2} h\right\|^{2}=\|A h\|^{2}$ for all $h \in \mathcal{D}(A)$.
} 
Corollary 3. Suppose $T$ is a positive operator in $\mathcal{H}$ and $\operatorname{Ext}(T)$ is nonempty. Then there exists a net $\left\{S_{\sigma}\right\}_{\sigma \in \Sigma} \subseteq \boldsymbol{B}(\mathcal{H})$ such that

(i) $S_{\sigma}$ is positive for all $\sigma \in \Sigma$,

(ii) $S_{\sigma} \leqslant S_{\tau} \prec T_{\mathrm{N}}$ for all $\sigma, \tau \in \Sigma$ such that $\sigma \leq \tau$,

(iii) $\operatorname{dim} \mathcal{R}\left(S_{\sigma}\right)<\infty$ for all $\sigma \in \Sigma$,

(iv) $T f=\lim _{\sigma \in \Sigma} S_{\sigma} f$ for all $f \in \mathcal{D}(T)$.

Before proving Corollary 3 , we formulate a useful lemma which is of independent interest (compare with [1, Corollary 3]; see also [12]).

Lemma 4. Suppose $T$ is a positive operator in $\mathcal{H}$ and $\operatorname{Ext}(T)$ is nonempty. If $\operatorname{dim} \mathcal{D}(T)<\infty$, then

(a) $T_{\mathrm{N}} \in \boldsymbol{B}(\mathcal{H})$,

(b) $\mathcal{R}\left(T_{\mathrm{N}}\right)=\mathcal{R}(T)$,

(c) $\operatorname{dim} \mathcal{R}\left(T_{\mathrm{N}}\right)=\operatorname{dim} \mathcal{D}(T)-\operatorname{dim} \mathcal{D}(T) \cap \mathcal{R}(T)^{\perp} \leqslant \operatorname{dim} \mathcal{D}(T)$.

Proof. It follows from $\operatorname{dim} \mathcal{D}(T)<\infty$ that $\operatorname{dim} \mathcal{R}(T)<\infty$ and $\mathcal{R}(T)$ is a closed linear subspace of $\mathcal{H}$. By [18, Theorem 2(v)], we have $\mathcal{N}\left(T_{\mathrm{N}}\right)=\mathcal{R}(T)^{\perp}$. Since the kernel of any selfadjoint operator reduces it, we obtain

$$
T_{\mathrm{N}}=A \oplus 0,
$$

where $A \in \boldsymbol{B}(\mathcal{R}(T))$ is positive and $\mathcal{N}(A)=\{0\}$. The injectivity of $A$ and $\operatorname{dim} \mathcal{R}(T)<\infty$ imply that $\mathcal{R}(A)=\mathcal{R}(T)$. This and (14) yield $\mathcal{R}\left(T_{\mathrm{N}}\right)=\mathcal{R}(A)=$ $\mathcal{R}(T)$. The condition (c) can be inferred from (b) and the equality $\mathcal{N}(T)=\mathcal{D}(T) \cap$ $\mathcal{R}(T)^{\perp}$ which in turn follows from $\mathcal{N}\left(T_{\mathrm{N}}\right)=\mathcal{R}(T)^{\perp}$.

Proof of Corollary 3, Denote by $\Sigma$ the set of all finite-dimensional linear subspaces of $\mathcal{D}(T)$ partially ordered by inclusion. For $\sigma \in \Sigma$, we set $S_{\sigma}=\left(\left.T\right|_{\sigma}\right)_{\mathrm{N}}$. By Lemma 4. the so-defined operator $S_{\sigma}$ is bounded, positive and of finite rank. If $\sigma, \tau \in \Sigma$ and $\sigma \subseteq \tau$, then $S_{\tau} \in \operatorname{Ext}\left(\left.T\right|_{\sigma}\right)$ and $T_{\mathrm{N}} \in \operatorname{Ext}\left(\left.T\right|_{\tau}\right)$. Applying Theorem 2, we obtain $S_{\sigma} \leqslant S_{\tau} \prec T_{\mathrm{N}}$. The condition (iv) is easily seen to be true 4

Remark 5. Note that Corollary 3 is equivalent to its particular version in which $T$ is assumed to be positive and selfadjoint (hence $T_{\mathrm{N}}=T$ ). Slightly modifying the proof of Corollary 3 , one can show that if $\mathcal{E}$ is a linear subspace of $\mathcal{D}(T)$ of linear dimension $\aleph_{0}$, then there exists a sequence $\left\{S_{n}\right\}_{n=1}^{\infty} \subseteq \boldsymbol{B}(\mathcal{H})$ of positive operators such that $S_{n} \leqslant S_{n+1} \prec T_{\mathrm{N}}, \operatorname{dim} \mathcal{R}\left(S_{n}\right) \leqslant n$ for all $n \geqslant 1$, and $T f=\lim _{n \rightarrow \infty} S_{n} f$ for all $f \in \mathcal{E}$. If $\mathcal{D}(T)$ is an infinite-dimensional linear subspace of a separable Hilbert space $\mathcal{H}$, then there always exists a linear subspace $\mathcal{E}$ of $\mathcal{D}(T)$ of linear dimension $\aleph_{0}$ which is a core for $T$, i.e. $T \subseteq \overline{\left.T\right|_{\mathcal{E}}}$, where $\overline{\left.T\right|_{\mathcal{E}}}$ is the closure of $\left.T\right|_{\mathcal{E}}$ (see the proof of [10, Proposition 4.1]). The interested reader is referred to the monograph [22] in which Stone worked out a different procedure for approximating a symmetric operator in an infinite-dimensional separable Hilbert space by a sequence of bounded selfadjoint operators; this approximation procedure enabled him to solve the extension problem in the case of densely defined semi-bounded operators (cf. [22, Theorems 5.1 and $9.21])$.

\footnotetext{
${ }^{4}$ Recall that $\left.T\right|_{\sigma} \subseteq S_{\sigma}$ and $\operatorname{dim} \mathcal{R}\left(S_{\sigma}\right) \leqslant \operatorname{dim} \sigma$ for all $\sigma \in \Sigma$.
} 
5.

The ensuing theorem is the main result of the paper.

Theorem 6. Suppose $T$ is a positive operator in $\mathcal{H}$ and $R \in \operatorname{Ext}(T)$. If $S$ is a positive selfadjoint operator in $\mathcal{H}$ satisfying the following three conditions:

(i) $\mathcal{D}(T) \subseteq \mathcal{D}\left(S^{1 / 2}\right)$,

(ii) $\left\|S^{1 / 2} g\right\|^{2} \leqslant\langle T g, g\rangle$ for all $g \in \mathcal{D}(T)$,

(iii) $R \prec S$,

then $S \in \operatorname{Ext}(T)$.

Proof. Define the map $\gamma: \mathcal{D}\left(S^{1 / 2}\right) \times \mathcal{D}\left(S^{1 / 2}\right) \rightarrow \mathbb{C}$ by

$$
\gamma(f, g)=\left\langle S^{1 / 2} f, S^{1 / 2} g\right\rangle-\left\langle R^{1 / 2} f, R^{1 / 2} g\right\rangle, \quad f, g \in \mathcal{D}\left(S^{1 / 2}\right) .
$$

According to (iii), $\gamma$ is a semi-inner product. By (i), (ii), (iii) and $T \subseteq R$, we have

$$
\left\|S^{1 / 2} g\right\|^{2} \leqslant\langle T g, g\rangle=\langle R g, g\rangle=\left\|R^{1 / 2} g\right\|^{2} \leqslant\left\|S^{1 / 2} g\right\|^{2}, \quad g \in \mathcal{D}(T),
$$

which gives us

$$
\gamma(g, g)=0, \quad g \in \mathcal{D}(T) .
$$

This and the Schwarz inequality applied to the semi-inner product $\gamma$ leads to

$$
|\gamma(f, g)|^{2} \leqslant \gamma(f, f) \gamma(g, g)=0, \quad f \in \mathcal{D}\left(S^{1 / 2}\right), g \in \mathcal{D}(T),
$$

which, together with $T \subseteq R$, yields

$$
\left\langle S^{1 / 2} f, S^{1 / 2} g\right\rangle=\left\langle R^{1 / 2} f, R^{1 / 2} g\right\rangle=\langle f, T g\rangle, \quad f \in \mathcal{D}\left(S^{1 / 2}\right), g \in \mathcal{D}(T) .
$$

Exploiting the fact that the operator $S^{1 / 2}$ is selfadjoint, we obtain

$$
\begin{aligned}
& \mathcal{D}(S)=\left\{g \in \mathcal{D}\left(S^{1 / 2}\right): S^{1 / 2} g \in \mathcal{D}\left(S^{1 / 2}\right)\right\} \\
& =\left\{g \in \mathcal{D}\left(S^{1 / 2}\right): \sup \left\{\left|\left\langle S^{1 / 2} f, S^{1 / 2} g\right\rangle\right|: f \in \mathcal{D}\left(S^{1 / 2}\right),\|f\| \leqslant 1\right\}<\infty\right\} .
\end{aligned}
$$

If $g \in \mathcal{D}(T)$, then (15) implies that

$$
\sup \left\{\left|\left\langle S^{1 / 2} f, S^{1 / 2} g\right\rangle\right|: f \in \mathcal{D}\left(S^{1 / 2}\right),\|f\| \leqslant 1\right\} \leqslant\|T g\| .
$$

This combined with (16) gives us the inclusion $\mathcal{D}(T) \subseteq \mathcal{D}(S)$. By the density of $\mathcal{D}\left(S^{1 / 2}\right)$ in $\mathcal{H}$, we deduce from (15) that $S g=T g$ for all $g \in \mathcal{D}(T)$, which completes the proof.

Corollary 7. Suppose $T$ is a positive operator in $\mathcal{H}$ and $\operatorname{Ext}(T)$ is nonempty. A positive selfadjoint operator $S$ in $\mathcal{H}$ extends $T$ if and only if the following three conditions hold:

(i) $\mathcal{D}(T) \subseteq \mathcal{D}\left(S^{1 / 2}\right)$,

(ii) $\left\|S^{1 / 2} g\right\|^{2} \leqslant\langle T g, g\rangle$ for all $g \in \mathcal{D}(T)$,

(iii) $T_{\mathrm{N}} \prec S$.

Proof. In view of Theorem 2, it is enough to show that conditions (i), (ii) and (iii) imply $T \subseteq S$. However this is a direct consequence of Theorem 6 .

Notice that Theorem 6 can be inferred form Theorem 2 and Corollary 7 . 
6.

Our next aim is to translate Corollary 7 into the language of quadratic forms. Given a positive selfadjoint operator $S$ in $\mathcal{H}$, we attach to it the closed densely defined positive quadratic form $\mathfrak{t}_{S}$ in $\mathcal{H}$ via

$$
\mathcal{D}\left(\mathfrak{t}_{S}\right)=\mathcal{D}\left(S^{1 / 2}\right) \text { and } \mathfrak{t}_{S}[f]=\left\|S^{1 / 2} f\right\|^{2} \text { for } f \in \mathcal{D}\left(\mathfrak{t}_{S}\right) .
$$

In view of (13), the above definition agrees with the one given in (2) in the case in which $T$ is a positive selfadjoint operator in $\mathcal{H}$. According to [11, Theorem 2.23, page 331], each closed densely defined positive quadratic form $\mathfrak{t}$ in $\mathcal{H}$ is associated with a (unique) positive selfadjoint operator $S$ in $\mathcal{H}$, i.e. $\mathfrak{t}=\mathfrak{t}_{S}$. Moreover, if $\mathfrak{t}_{1}$ and $\mathfrak{t}_{2}$ are two such forms and $S_{1}$ and $S_{2}$ are corresponding positive selfadjoint operators, then $\mathfrak{t}_{1} \prec \mathfrak{t}_{2}$ if and only if $S_{1} \prec S_{2}$.

Corollary 8. If $T$ is a positive operator in $\mathcal{H}$ and $\mathfrak{t}$ is a closed densely defined positive quadratic form in $\mathcal{H}$, then the following conditions are equivalent:

(i) there exists $S \in \operatorname{Ext}(T)$ such that $\mathfrak{t}_{S}=\mathfrak{t}$,

(ii) $\mathcal{D}(T) \subseteq \mathcal{D}(\mathfrak{t}), \mathfrak{t}[g] \leqslant\langle T g, g\rangle$ for all $g \in \mathcal{D}(T)$ and $\mathfrak{t}_{T} \prec \mathfrak{t}$, where $\mathfrak{t}_{T}$ is as in (2).

Proof. Since $\mathfrak{t}_{T} \prec \mathfrak{t}$ implies $\overline{\mathcal{D}\left(\mathfrak{t}_{T}\right)}=\mathcal{H}$, Corollary 8 follows from Theorem 2 and Corollary 7.

7.

M. G. Krein gave a complete characterization of the class $\operatorname{Ext}(T)$ in the case in which $T$ is densely defined (cf. [12, 15]; see also [6, 3, 4, for further investigation in this direction). The next theorem extends this characterization to the case in which $T$ is not assumed to be densely defined.

Theorem 9. Let $T$ be a positive operator in $\mathcal{H}$. If $R, Q \in \operatorname{Ext}(T)$ and $S$ is a positive selfadjoint operator in $\mathcal{H}$ such that $R \prec S \prec Q$, then $S \in \operatorname{Ext}(T)$.

Proof. Since

$$
\mathcal{D}(T) \subseteq \mathcal{D}(Q) \subseteq \mathcal{D}\left(Q^{1 / 2}\right) \subseteq \mathcal{D}\left(S^{1 / 2}\right)
$$

and

$$
\left\|S^{1 / 2} g\right\|^{2} \leqslant\left\|Q^{1 / 2} g\right\|^{2}=\langle Q g, g\rangle=\langle T g, g\rangle, \quad g \in \mathcal{D}(T),
$$

we can apply Theorem 6 to complete the proof.

The ensuing corollary is a consequence of Theorems 2 and 9 , On the other hand, Theorem 9 follows from Theorem 2 and Corollary 10 .

Corollary 10. Suppose $T$ is a positive operator in $\mathcal{H}$ and $\operatorname{Ext}(T)$ is nonempty. $A$ positive selfadjoint operator $S$ in $\mathcal{H}$ belongs to $\operatorname{Ext}(T)$ if and only if there exists $Q \in \operatorname{Ext}(T)$ such that $T_{\mathrm{N}} \prec S \prec Q$.

\footnotetext{
${ }^{5}$ Recall that for positive quadratic forms $\mathfrak{t}_{1}$ and $\mathfrak{t}_{2}$ in $\mathcal{H}$, the order relation $\mathfrak{t}_{1} \prec \mathfrak{t}_{2}$ is understood to mean that $\mathcal{D}\left(\mathfrak{t}_{2}\right) \subseteq \mathcal{D}\left(\mathfrak{t}_{1}\right)$ and $\mathfrak{t}_{1}[g] \leqslant \mathfrak{t}_{2}[g]$ for all $g \in \mathcal{D}\left(\mathfrak{t}_{2}\right)$ (cf. [11 page 330]).
} 
If $T$ is a densely defined positive operator in $\mathcal{H}$, then $\operatorname{Ext}(T)$ is nonempty and there exists the largest member $T_{\mathrm{F}}$ of $\operatorname{Ext}(T)$ with respect to the partial order $\prec$. $T_{\mathrm{F}}$ is called the Friedrichs extension of $T$ (cf. [9, 8, 11, 23]; see also [15] for historical comments). We conclude this paper with a result which is due to M. G. Krein [12]. It is an immediate consequence of Corollary 10.

Corollary 11. Let $T$ be a densely defined positive operator in $\mathcal{H}$. Then

$$
\operatorname{Ext}(T)=\left\{S: S \text { is a positive selfadjoint operator in } \mathcal{H} \text { such that } T_{\mathrm{N}} \prec S \prec T_{\mathrm{F}}\right\} \text {. }
$$

\section{ACKNOWLEDGEMENT}

The authors would like to thank the referee for helpful suggestions, which have helped to improve the final version of the paper.

\section{REFERENCES}

[1] T. Ando, K. Nishio, Positive self-adjoint extensions of positive symmetric operators, Tôhoku Math. J. 22 (1970), 57-65. MR0264422 (41:9016)

[2] Yu. M. Arlinskiı̌, S. Hassi, Z. Sebestyén, H. S. V. de Snoo, On the class of extremal extensions of a nonnegative operator, Operator Theory: Adv. Appl. 127, Birkhäuser, Basel, 2001, 41-81. MR1902794 (2003d:47028)

[3] Yu. Arlinskiü, E. Tsekanovskiŭ, On von Neumann's problem in extension theory of nonnegative operators, Proc. Amer. Math. Soc. 131 (2003), 3143-3154. MR.1992855 (2004h:47034)

[4] Yu. Arlinskiı̌, E. Tsekanovskiı̌, The von Neumann problem for nonnegative symmetric operators, Integral Equations Operator Theory 51 (2005), 319-356. MR2126815

[5] T. Ja. Azizov, I. S. Iohvidov, V. A. Straus, The normally solvable extensions of a closed symmetric operator (Russian), Mat. Issled. 7 (1972), 16-26. MR0308842 (46:7954)

[6] M. Š. Birman, On the theory of self-adjoint extensions of positive definite operators (Russian), Mat. Sb. N.S. 38 (1956), 431-450. MR.0080271(18:220d)

[7] M. Sh. Birman, M. Z. Solomjak, Spectral theory of selfadjoint operators in Hilbert space, D. Reidel Publishing Co., Dordrecht, 1987. MR1192782 (93g:47001)

[8] H. Freudenthal, Über die Friedrichssche Fortsetzung halbbeschränkter Operatoren, Akademie van Wetenschappente Amsterdam, Proceedings, ser. A, 39 (1936), 832-833.

[9] K. Friedrichs, Spektraltheorie halbbeschränkter Operatoren und Anwendungen auf die Spektralzerlegung von Differenzialoperatoren, Math. Ann. 109 (1934), 465-485. MR.1512905

[10] J. Janas, J. Stochel, Selfadjoint operator matrices with finite rows, Ann. Polon. Math. 66 (1997), 155-172. MR1438336 (97k:47023)

[11] T. Kato, Perturbation theory for linear operators, Springer-Verlag, Berlin, 1980. MR1335452 (96a:47025)

[12] M. G. Krein, The theory of self-adjoint extensions of semi-bounded Hermitian transformations and its applications. I (Russian), Mat. Sb. 20 (1947), 431-495. MR0024574 (9:515c)

[13] V. Prokaj, Z. Sebestyén, On extremal positive operator extensions, Acta Sci. Math. (Szeged), 62 (1996), 485-491. MR:1430868 (97m:47012)

[14] V. Prokaj, Z. Sebestyén, On Friedrichs extensions of operators, Acta Sci. Math. (Szeged), 62 (1996), 243-246. MR1412931 (97i:47040)

[15] F. Riesz, B. Sz.-Nagy, Leçons d'analyse fonctionnelle, Akadémiai Kiadó, Budapest, 1972. MR0179567 (31:3815)

[16] Z. Sebestyén, Restrictions of positive operators, Acta Sci. Math. (Szeged) 46 (1983), 299-301. MR0739047 (85i:47003b)

[17] Z. Sebestyén, Positivity of operator products, Acta Sci. Math. (Szeged) 66 (2000), 287-294. MR1768867 (2001b:47004)

[18] Z. Sebestyén, E. Sikolya, Krein-von Neumann and Friedrichs extensions, Acta Sci. Math. (Szeged) 69 (2003), 323-336. MR1991670 (2004d:47055)

[19] Z. Sebestyén, J. Stochel, Restrictions of positive self-adjoint operators, Acta Sci. Math. (Szeged) 55 (1991), 149-154. MR1124953(92i:47024)

[20] Z. Sebestyén, J. Stochel, On products of unbounded operators, Acta Math. Hungar. 100 (2003), 105-129. MR1984863 (2004c:47003) 
[21] Z. Sebestyén, J. Stochel, Reflection symmetry and symmetrizability of Hilbert space operators, Proc. Amer. Math. Soc. 133 (2004), 1727-1731. MR2120258

[22] M. H. Stone, Linear transformations in Hilbert space and their applications to analysis, Amer. Math. Soc. Colloq. Publ. 15, Amer. Math. Soc., Providence, R.I., MR1451877 (99k:47001)

[23] J. Weidmann, Linear operators in Hilbert spaces, Springer-Verlag, Berlin, Heidelberg, New York, 1980. MR0566954(81e:47001)

[24] J. von Neumann, Allgemeine Eigenwerttheorie Hermitischer Funktionaloperatoren, Math. Annalen 102 (1929), 49-131.

Department of Applied Analysis, Eötvös L. University, Pázmány Péter sétány 1/C., BudAPEST H-1117, Hungary

E-mail address: sebesty@cs.elte.hu

Instytut Matematyki, Uniwersytet Jagielloński, ul. Reymonta 4, PL-30059 Kraków, POLAND

E-mail address: stochel@im.uj.edu.pl 